\title{
Hilfebedarfe pflegender Angehöriger von onkologischen Patienten im Raum Erfurt
}

\author{
Matthias Drossel ${ }^{1,2} \cdot$ Daniela Sperling ${ }^{3}$ \\ Eingegangen: 24. Januar 2021 / Angenommen: 15. November 2021 / Online publiziert: 5. Januar 2022 \\ (c) Der/die Autor(en) 2021
}

\section{Zusammenfassung}

Durch die zunehmende Ambulantisierung in der Krebstherapie steigen die Anforderungen an pflegende Angehörige, die den onkologisch Erkrankten unterstützend zur Seite stehen. Die Belastungen, die diese als informell Pflegende im Laufe der Pflegezeit empfinden, verstärken sich über die Dauer. Spezielle Unterstützungs- und Hilfsangebote können diese Belastungen reduzieren. Das Ziel der Untersuchung ist, Hilfebedarfe pflegender Angehöriger speziell im Raum Erfurt zu ermitteln und das bereits bestehende Angebotsspektrum in diesem Gebiet darzustellen. Dazu wurden in zwei Forschungsschritten Experten und informell Pflegende mithilfe leitfadengestützter Interviews befragt. Hierbei zeigte sich ein Defizit in der Verfügbarkeit von Hilfsangeboten, die speziell an informell Pflegende gerichtet sind. Die informell Pflegenden sind jedoch nur selten über Unterstützungsmöglichkeiten informiert. Aus diesem Grund muss die informelle Pflege im onkologischen Bereich durch gezielte, frühzeitige Information und Beratung über Unterstützungsleistungen und eine Erweiterung des Angebotsspektrums gestärkt werden.

Schlüsselwörter Pflegende Angehörige $\cdot$ Informelle Pflege $\cdot$ Onkologie $\cdot$ Erfurt $\cdot$ Hilfebedarfe

\section{Need of support for informal carers of oncological patients in the Erfurt area}

\begin{abstract}
Due to the increasing outpatient treatment in cancer therapy, the demands on caring relatives are increasing. The stress that informal carers feel over the course of the care period increases the longer it lasts. Special offers of help could make a difference. The aim of this study was to determine the need of support for caring relatives especially in the Erfurt area and to present the range of offers already available in this area. For this purpose, experts and informal carers were interviewed in two research steps. It became apparent that there is a deficit in the availability of help offers for informal carers. In addition, family carers are rarely informed about such support services. For this reason, informal care in the oncological sector must be strengthened through targeted early information about support services and an expansion of the range of services offered.
\end{abstract}

Keywords Caring relatives $\cdot$ Informal care $\cdot$ Oncology $\cdot$ Erfurt $\cdot$ Support needs

Matthias Drossel

matthias.drossel@srh.de

$\triangle$ Daniela Sperling

dsperling@kkh-erfurt.de

1 SRH Hochschule für Gesundheit Gera, Gera, Deutschland

2 Bamberger Akademien für Gesundheits- und Pflegeberufe, Bamberg, Deutschland

3 Schule für Pflege, Krankenhaus St. Nepomuk Erfurt, Erfurt, Deutschland

\section{Hintergrund und Problemstellung}

Die Diagnose bösartige Tumorerkrankung wurde im Jahr 2013 über 482.000 Menschen in Deutschland mitgeteilt. Die Zahl der Neuerkrankungen steigt seit Beginn der kontinuierlichen Registrierung von Krebserkrankungen stetig an (Deutsches Krebsforschungszentrum 2017; Robert KochInstitut 2016). Demografische Entwicklungen und hohe Alterserwartungen, aber auch neue Screeningmethoden sind Gründe für die steigenden Zahlen von diagnostizierten Neu- 
erkrankungen. Die Daten zur Registrierung von bösartigen Neubildungen werden im Freistaat Thüringen in den 5 verschiedenen Tumorzentren erfasst (Göbel 2017). Die Stadt Erfurt registriert die höchste Zahl an Neuerkrankungen im Freistaat. So werden $40 \%$ aller Tumorneuregistrierungen in Erfurt registriert. Es zeigt sich, dass seit 1993 Neuregistrierungen stetig ansteigen. Die Folge ist, dass immer mehr Menschen mit einer Tumorerkrankung konfrontiert werden (Göbel 2017).

Eine Krebsdiagnose ist für die Betroffenen und deren Angehörige eine Krisenphase. Dieser Einschnitt in das Leben des Erkrankten, aber auch der Angehörigen und der sozialen Netzwerke, hat eine große Bedeutung und Tragweite (Huse-Kleinstoll 2013). Sie wollen den Betroffenen bei der Krankheitsbewältigung und während der Therapieversuche, z. B. Chemo- oder Strahlentherapie, unterstützen und gehen dabei oft über ihre eigenen Grenzen hinaus. Daraus resultieren meist große Belastungen für die informell Pflegenden. So leidet circa ein Drittel der Angehörigen unter Stresssymptomen oder Verhaltensstörungen (Weihs und Reiss 2000). Götze et al. (2015) beschreiben die fortschreitenden Belastungen und die daraus resultierende Erschöpfung der pflegenden Angehörigen. Sie stiegen bei den informell Pflegenden im Laufe des zweijährigen Befragungszeitraumes signifikant an. Aufgrund der ermittelten Ergebnisse empfehlen Götze et al. (2015) eine frühzeitige Einbindung Angehöriger in psychoonkologische Unterstützungsangebote oder eine Kontaktaufnahme zu Krebsberatungsstellen. Des Weiteren wird eine fachliche Beihilfe, z.B. Anleitung zu Pflegemaßnahmen, als Hilfsangebot empfohlen, um Unsicherheiten und körperliche Belastungen abzubauen (Götze et al. 2015). Rosenberger et al. (2012) beschreiben mögliche Hilfsangebote. 132 Angehörige und 362 Krebspatienten wurden befragt. Es erfolgte eine Erhebung der Belastungssituation mittels Fragebogen. In einem darauffolgenden Gespräch mit einem Therapeuten wurden Hilfebedarfe der Betroffenen ermittelt. Der größte Bedarf bestand im psychologischen Bereich. Am zweithäufigsten wurde das Informationsbedürfnis bezüglich des Gesundheitssystems und seiner Leistungen genannt (Rosenberger et al. 2012). Die Autoren fordern außerdem mehr Transparenz und Öffentlichkeitsarbeit bezüglich des Angebotsspektrums der Spezialambulanz, da dieses vielen Befragten nicht bekannt war (Rosenberger et al. 2012). Es zeigte sich auch, dass nur ein Viertel der informell Pflegenden die Ambulanz aus eigenem Antrieb aufsucht, oder aufgrund der Empfehlung von Familienmitgliedern oder Ärzten (Rosenberger et al. 2012).

Drossel (2018) untersuchte Hilfe- und Unterstützungsbedarfe informell Pflegender. Ziel dieser Untersuchung war es, ein Handlungskonzept zur Unterstützung der informellen Pflege in dieser Region zu entwickeln und diesen Bereich zu stärken (Drossel 2018). Drossel (2018) fordert, dass eine Sensibilisierung auf pflegende Angehörige und diejenigen, die sie versorgen, in verschiedenen Teilbereichen erfolgen muss und so besser auf deren Probleme eingegangen und ihnen der Pflegealltag erleichtert wird (Drossel 2018). Auch hier ist das Angebot von Informationen, Beratung und Anleitung, z.B. in Form von aufsuchender Pflegeberatung, Familiengesundheitspflege und Gemeindepflege oder in Pflegestützpunkten, als wichtiges Ergebnis formuliert. Des Weiteren zeigen die Ergebnisse auf, dass eine Engagement-Gesellschaft aufgebaut werden muss. Diese ist ein wichtiges Mittel zur Stärkung informell Pflegender, denn durch die aktive Gestaltung und Teilhabe aller Mitglieder einer Gemeinde werden soziale Netzwerke gestärkt und wirken unterstützend für die informell Pflegenden (Drossel 2018).

Eine weitere Unterstützung für Betroffene und Angehörige stellt die Psychoonkologie dar. Sie hilft bei der Krankheitsbewältigung, aber auch bei psychischen Problemen, die infolge der Therapie auftreten (Deutsche Krebsgesellschaft 2014). Die S3-Leitlinie zu psychoonkologischer Beratung und Diagnostik empfiehlt, dass die Hilfsangebote kostenlos, kurzfristig verfügbar und niederschwellig in psychotherapeutischen Praxen sowie in psychosozialen Krebsberatungsstellen bereitgestellt werden sollen. Die Versorgungsstruktur im Rahmen der Psychoonkologie hat sich positiv entwickelt, dennoch muss an dem Aufbau eines sektorenübergreifenden und interprofessionellen Netzwerks gearbeitet werden, um eine flächendeckende Weiterbetreuung vom stationären in den ambulanten Bereich zu gewährleisten (Rosenberger 2017). Dies gewinnt zunehmend an Bedeutung, da die Einbindung pflegender Angehöriger in das interprofessionelle therapeutische Team durch die Ambulantisierung in der Onkologie wichtiger wird.

Die Versorgung onkologischer Erkrankungen erfolgt in Deutschland innerhalb eines dreistufigen Zentrenmodells (siehe Abb. 1). Die Organkrebszentren bilden die Basis dieses Modells. Hier erfolgt die Behandlung des Tumor-

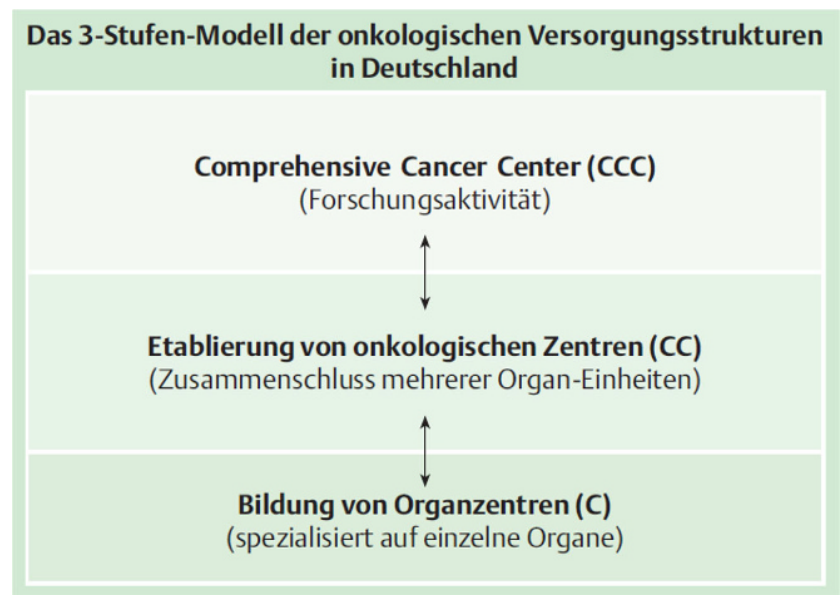

Abb. 1 Zentrenmodell der onkologischen Versorgung (Aus Beckmann et al. 2008, S. 661) 
leidens organspezifisch. Der Zusammenschluss mehrerer Organkrebszentren unter Mitwirkung organübergreifender Fachdisziplinen, z. B. Radiologie und Radioonkologie, Psychoonkologie und Palliativmedizin, wird onkologisches Zentrum oder auch Cancer Center genannt. Hier erfolgen außerdem Tumordokumentation und Tumorkonferenzen. Ambulante und stationär oder teilstationäre Dienstleister, z.B. Arztpraxen, medizinische Versorgungszentren und Krankenhäuser, müssen hier zusammenarbeiten. Ein Comprehensive Care Center ist die Versorgungsstruktur mit der komplexesten medizinisch-pflegerischen Versorgung. Dies Zentren sind zusätzlich noch in der Forschung tätig (Beckmann et al. 2008).

Diagnostiziert wird eine Tumorerkrankung zumeist an einem onkologischen Zentrum (Beckmann et al. 2008). Die therapeutische Weiterversorgung des Patienten kann in der Regel dort erfolgen. Es ist zu beobachten, dass sich diese Leistungen zunehmend auch in den ambulanten Sektor verlagern (Stoll und Schmidt 2011). Zur Gewährleistung einer umfassenden Weiterversorgung der Betroffenen werden Unterstützungsdienste aus dem Homecare-Konzept hinzugezogen. Es grenzt sich von der häuslichen Pflege ab und ermöglicht verordnete therapeutische Leistungen im eigenen Umfeld (von Reibnitz 2008).

Regionale Unterschiede in den Versorgungsstrukturen zeigen, dass weitere Forschungen notwendig sind, um die Ambulantisierungsprozesse erfolgreich zu gestalten. Diese Besonderheiten und deren spezifische Anforderungen müssen aus Sicht der Autoren weiter analysiert werden und Lösungsansätze erarbeitet werden.

\section{Zielstellung und Forschungsfragen}

Ziel der Untersuchung ist es, die Situation informell Pflegender, die im Raum Erfurt onkologische Patienten betreuen, darzustellen. Als forschungsleitend galt folgende Fragestellung: Welche Unterstützungsangebote beschreiben informell Pflegende onkologischer Patienten im Raum Erfurt, die dazu beitragen, diese Pflege zu Hause längerfristig gestalten zu können? Zur Beantwortung dieser Frage ist es wichtig, weitere Aspekte zu berücksichtigen. Es sollen bestehende Hilfsangebote im Raum Erfurt für pflegende Angehörige onkologischer Patienten erkannt werden, und ob die informell Pflegenden über das bestehende Angebotsspektrum informiert sind und dieses wahrnehmen. Auch muss der subjektive Unterstützungs- und Hilfebedarf, den die pflegenden Angehörigen verspüren, erfragt werden. Der Begriff informell Pflegende ist in der Literatur nicht einheitlich verwendet. Die Autoren orientieren sich an der Begriffsabgrenzung von Drossel (2018): „Informell Pflegende sind Personen, die ohne Vergütung gemäß einer Anstellung durch den Pflegebedürftigen oder dessen Angehörige und ohne professionelle Ausbildung Menschen in ihrem häuslichen Umfeld bei der Verrichtung von pflegerischen und/ oder hauswirtschaftlichen Tätigkeiten aufgrund einer Einschränkung in den alltäglichen Dingen des Lebens (ATL) unterstützen. Beispiele hierfür sind: Familienangehörige, Nachbarn, Ehrenamtliche etc."

\section{Methode}

Die Datenerhebung erfolgte im Zeitraum von August 2018 bis Januar 2019 in einem zweischrittigen Forschungsprozess. Zur Bearbeitung der Forschungsfragen wurde ein qualitatives Design gewählt, da Einzelfälle und deren subjektive Einstellungen, Meinungen und Bedürfnisse durch einen qualitativen Forschungsprozess zielführend elaboriert werden. Diese Beschreibungen und deren Auswertungen folgen einem induktiven Forschungsvorgehen (Corbin und Hildebrand 2003). Zur Annäherung an das Forschungsfeld war es notwendig, in einem ersten Forschungsschritt den Leistungsumfang von Unterstützungsangeboten für pflegende Angehörige von onkologischen Patienten im Raum Erfurt und ihre Annahme durch die Betroffenen zu ermitteln. Angelehnt an die Methode eines Leitfadeninterviews nach Helfferich (2009) fanden zu diese Zweck Gespräche mit 5 ausgewählten Experten statt. „Ob jemand als Expertin angesprochen wird, ist in erster Linie abhängig vom jeweiligen Forschungsinteresse. Expertin ist ein relationaler Status. [...] Der ExpertInnenstatus wird in gewisser Weise vom Forscher verliehen, begrenzt auf eine spezifische Fragestellung“" (Meuser und Nagel 1991, S. 443). Die Auswahl der Interviewpartner und Titulierung als Experten erfolgen durch die Forscher.

Die Ergebnisse des ersten Forschungsschrittes wurden mithilfe der „SPSS-Methode“ nach Helfferich (2009) als Grundlage für einen Interviewleitfaden genutzt. Ziel des zweiten Forschungsschrittes war es, das Belastungserleben und die daraus resultierenden Hilfebedarfe informell Pflegender von onkologischen Patienten im Raum Erfurt fokussiert darzustellen. Es wurden halbstandardisierte Interviews mit informell Pflegenden, die onkologische Patienten in der eigenen Häuslichkeit betreuen, geführt. Es erfolgten $\mathrm{Au}-$ dioaufzeichnungen der Gespräche, ein Postmemo und eine zeitnahe Transkription, angelehnt an die Regeln von Dresing und Pehl (2018). Die Transkripte wurden anschließend nach den 7 Schritten der qualitativen Inhaltsanalyse nach Kuckartz (2012) softwaregestützt ausgewertet.

\section{Forschungsraum}

Die Daten zur Registrierung von bösartigen Neubildungen werden in Thüringen in den 5 verschiedenen Tumorzen- 
tren Erfurt, Gera, Suhl, Jena und Nordhausen erfasst. Erfurt, als größte Stadt Thüringens, hat die höchste Zahl an Neuerkrankungen. In Erfurt werden $40 \%$ aller Tumorneuregistrierungen registriert. Es zeigt sich, dass die Zahl an neu diagnostizierten Tumoren seit Beginn der Erfassung gestiegen ist. Infolgedessen rücken Krebserkrankungen immer mehr in den Fokus. Immer mehr Menschen und deren Zugehörige müssen sich mit der Diagnose und ihren Folgen auseinandersetzen. Die Versorgung von onkologischen Erkrankungen in der Region Erfurt wird in Zentren der Stufen 1 (Organkrebszentrum) und 2 (onkologisches Zentrum) geleistet. Organkrebszentren gibt es in Arztpraxen und in den beiden Krankenhäusern der Stadt.

\section{Ergebnisse des ersten Forschungsschrittes}

Mit 5 Experten wurden Interviews geführt. Diese waren teilstrukturiert, und deren Ergebnisse dienen als Grundlage für die Erstellung eines Leitfadens für den zweiten Forschungsschritt. Einschlusskriterien bei der Auswahl des Samples waren, dass die Experten sich in ihrer Tätigkeit im Raum Erfurt mit onkologischen Patienten und deren

Tab. 1 Ergebnisse der Expertengespräche

\begin{tabular}{lll}
\hline Kategorien & Beschreibung & Kurzinhalte \\
\hline Entwicklung & Umfasst alle Aussagen & Beratungsstelle für Geschwulstkranke und ältere Menschen gibt es seit DDR-Zeit; Beratungsbe- \\
der & zur Etablierung der in & darf steigt stetig, weitere Stellen wären notwendig \\
Hilfsangebote & Erfurt ansässigen & Selbsthilfegruppe (SHG) Prostatakrebs richtet sich vorrangig an Betroffene, wird vom Bundes- \\
in Erfurt & Hilfsangebote & verband
\end{tabular}

in Erfurt Hilfsangebote verband unterstützt

SHG ,pflegende Angehörige“ wurde aus eigener Betroffenheit gegründet, richtet sich an Angehörige, die Familienmitglieder mit Demenz, M. Parkinson und Osteoporose versorgen

Pflegeüberleitung seit 10 Jahren im Krankenhaus tätig; auch sie verzeichnet eine Bedarfssteigerung

Kontaktstelle für Selbsthilfegruppen (KISS) stellt in letzten 30 Jahren Wandel von somatischen hin zu psychiatrischen Gruppen, häufig auch nur Betroffenengruppen, fest

\begin{tabular}{|c|c|c|}
\hline $\begin{array}{l}\text { Informations- } \\
\text { quellen und } \\
\text { Kontaktauf- } \\
\text { nahme }\end{array}$ & $\begin{array}{l}\text { Thematisiert die von } \\
\text { Interessierten genutzten } \\
\text { Möglichkeiten, um zu } \\
\text { den Angeboten Kontakt } \\
\text { aufzunehmen }\end{array}$ & $\begin{array}{l}\text { Am häufigsten und durch alle Altersgruppen hindurch erfolgt die Kontaktaufnahme telefonisch } \\
\text { Informationen bekommen jüngere Interessierte aus dem Internet } \\
\text { Netzwerkarbeit in den Krankenhäusern und das Verteilen von Flyern stellen auch eine Informati- } \\
\text { onsquelle dar }\end{array}$ \\
\hline Arbeitsweise & $\begin{array}{l}\text { Vereint Aussagen, die } \\
\text { zur Arbeit in den } \\
\text { vermittelnden Stellen } \\
\text { und der Beratungsstelle } \\
\text { getroffen werden }\end{array}$ & $\begin{array}{l}\text { Beratungsstelle hat feste Sprechzeiten, macht Termine nur in Ausnahmefällen, Wartezeiten kön- } \\
\text { nen für alle Klienten überschaubar gehalten werden } \\
\text { Klienten sind zumeist Betroffene } \\
\text { Pflegeüberleitung kann nur während des Krankenhausaufenthalts und kurz danach aktiv sein, } \\
\text { Erarbeitung eines Versorgungskonzepts gemeinsam mit onkologischer Fachschwester } \\
\text { KISS vermittelt Kontaktdaten von Selbsthilfegruppen an Interessierte, prüft, ob ehrliches Interes- } \\
\text { se an einem Hilfsangebot besteht }\end{array}$ \\
\hline Themen & $\begin{array}{l}\text { Experten beschreiben } \\
\text { hier die in ihren } \\
\text { Angeboten } \\
\text { angesprochenen } \\
\text { Themen und Prozesse } \\
\text { zur Themenfindung }\end{array}$ & $\begin{array}{l}\text { Leiterin der Beratungsstelle beschreibt Wandel der besprochenen Themen von anfänglich organi- } \\
\text { satorischen Problematiken hin zu persönlichen Problemen und Ängsten } \\
\text { SHG ,pflegende Angehörige“ hat festen Jahresplan, in dem Themen und Gastreferenten festge- } \\
\text { legt sind } \\
\text { Leiter der Gruppe Prostatakrebs empfindet Themenfindung in seiner Gruppe als schwierig, be- } \\
\text { dingt durch stetigen Teilnehmerwechsel }\end{array}$ \\
\hline $\begin{array}{l}\text { Dynamiken } \\
\text { der SHG }\end{array}$ & $\begin{array}{l}\text { Vereint Aussagen der } \\
\text { Selbsthilfegruppenleiter } \\
\text { zur Charakteristik ihrer } \\
\text { Gruppen }\end{array}$ & $\begin{array}{l}\text { SHG ,pflegende Angehörige“ trifft sich monatlich in einem Stadtteilzentrum } \\
\text { Probleme der Gruppe sind Finanzierung und Überalterung der Gruppenmitglieder } \\
\text { Dennoch erreichen Leiter gehäuft Beratungsgesuche von anderen informell Pflegenden } \\
\text { Bei SHG Prostatakrebs stellt Mitgliederwechsel, bedingt durch Alter, Genesung, aber auch } \\
\text { Versterben ein Problem dar } \\
\text { Finanziert wird die Gruppe vom Bundesverband Prostatakrebs e. V. }\end{array}$ \\
\hline $\begin{array}{l}\text { Angebots- } \\
\text { spektrum }\end{array}$ & $\begin{array}{l}\text { Umfasst Aussagen der } \\
\text { Experten zu den } \\
\text { Angebotsmöglichkeiten } \\
\text { in Erfurt und dem } \\
\text { Annahmeverhalten } \\
\text { durch Angehörige }\end{array}$ & $\begin{array}{l}\text { Angebotsstruktur in Erfurt für informell Pflegende onkologischer Patienten ist defizitär } \\
\text { Das Fehlen eines Pflegestützpunkts macht die Informationsbeschaffung schwierig und unüber- } \\
\text { sichtlich, funktionierendes soziales Netzwerk ist essenziell } \\
\text { Hilfebedarf wird von den Angehörigen häufig nicht als solcher wahrgenommen und verdrängt, } \\
\text { Betroffener steht im Fokus der Hilfesuche } \\
\text { Teilnahme an SHG für Angehörige nur mit Einschränkungen möglich, da diese Betroffenengrup- } \\
\text { pen sind }\end{array}$ \\
\hline
\end{tabular}


pflegerische Themen auseinandersetzen, z. B. informierend, beratend und schulend. Die 5 Interviews fanden in einem Zeitraum im August bis September 2018 statt. Sie wurden am Arbeitsplatz des jeweiligen Experten geführt oder bei diesen zu Hause. Daraufhin wurden theoretische Beschreibungen auf der Basis von Tonaufzeichnungen und Gedächtnisprotokollen angefertigt. Unter Hinzunahme der qualitativen Inhaltsanalyse, angelehnt an Mayring (2015), fand eine Paraphrasierung relevanter Passagen der Protokollierungen und Transkripte statt. Diese wurden durch die Hinzunahme der von Mayring in diesem Zusammenhang festgelegten Interpretationsregeln auf ein höheres Abstraktionsniveau gebracht, aus dem sich dann Kategorien als Basis für den Interviewleitfaden für den Forschungsschritt 2 elaborieren ließen. Die Ergebnisse werden in Tab. 1 dargestellt.

\section{Ergebnisse des zweiten Forschungsschrittes}

Im zweiten Forschungsschritt wurden 5 informell Pflegende in halbstrukturierten Leitfadeninterviews (Helfferich 2009) befragt. Als Einschlusskriterium für die Auswahl der Interviewpartner wurde festgelegt, dass diese einen Angehörigen mit einer Tumorerkrankung in der eigenen Häuslichkeit unterstützen und/oder versorgen. Der Wohnsitz der Pflegenden sollte in Erfurt oder in einem Umkreis bis zu $30 \mathrm{~km}$ sein. Es erfolgte ein Einschluss von kurativen oder palliativen Behandlungen der Krebserkrankung, sowohl in der stationären als auch in der klinischen Versorgung. Die Rekrutierung des Samples erfolgte über berufliche Netzwerke der Forscher. Es wurden 3 Männern und 2 Frauen im Alter von 48 bis 73 Jahren ausgewählt. Nur ein informell Pflegender war neben der Pflegetätigkeit noch berufstätig; die anderen befanden sich bereits im Ruhestand. Die zu Pflegenden waren meist die Ehepartner der Befragten; nur in einem Fall war es die Mutter, die an Krebs erkrankt war und versorgt wurde.

Die Kontaktaufnahme erfolgte zunächst telefonisch. Vor dem verabredeten Gesprächstermin erhielten die beteiligten Angehörigen einen Informationsbogen zum Forschungsvorhaben. Die Teilnehmer der Untersuchung hatten die Möglichkeit, sich umfassend über die Zielsetzung der Arbeit zu informieren und mithilfe der beigefügten Einverständniserklärung ihre informierte Einwilligung zur Studienteilnahme zu geben. Die Befragten gelten als nichtvulnerable Befragungsgruppe. Eine ethisch würdige Grundhaltung der Forscher galt als grundlegend. Die Datenerhebung erfolgte von Dezember 2018 bis Januar 2019. Jedes Interview wurde durch eine Audioaufzeichnung begleitet, und es folgte eine zeitnahe Transkription nach den Regeln von Dresing und Pehl (Dresing und Pehl 2018). Die Transkripte wurden anschließend nach den 7 Schritten der qualitativen Inhaltsanalyse nach Kuckartz (2012) ausgewertet. Mit dieser
Methode konnten 6 Hauptkategorien mit durchschnittlich 3 Subkategorien generiert werden. Drei ausgewählte Kategorien werden im Folgenden beschrieben.

\section{Kategorie Informationsquellen}

Die informell Pflegenden beschrieben, dass sie hilfreiche Informationen zu Unterstützungsleistungen und Hilfen überwiegend über den informellen Weg bekamen, z. B. im eigenen sozialen Umfeld, durch Mitpatienten der Pflegeempfänger, das Internet oder andere informelle Netzwerke. Weitere Informationen erhalten diese über Krankenkassen oder auch durch Pflegedienste und SAPV-Teams.

\section{Kategorie subjektive Bedarfe an Hilfeangeboten}

Nur eine Befragte benennt präzise Bedarfe an Unterstützungsleistungen. Sie beschreibt, dass Selbsthilfegruppen als Austausch ideal wären. Jedoch wohnt sie in einem ländlichen Bereich. Dies gestaltet die Teilhabe aufgrund mangelnder Mobilität aus ihrer Sicht schwierig. Ein weiterer Wunsch ist die seelsorgerischer Unterstützung. Zwei weitere Personen beschreiben, dass sie Hilfsangebote nur annehmen, wenn die Empfehlung von einem Arzt kommt. Die Eigenmotivation, ein Unterstützungsangebot für sich zu nutzen, besteht nicht. Eine andere informell Pflegende berichtet hingegen, dass die Suche und Annahme von Unterstützungsleistungen für sie zumeist an eine labile Persönlichkeitsstruktur, also Schwäche, geknüpft ist. Für sich selbst lehnt sie Unterstützungsleistungen ab. Die Befragten beschreiben, dass Sie die Anforderungen alleine bewältigen möchten und Dritte ungerne in die Problematik miteinbeziehen. Für einen Gesprächspartner spielt auch das soziale Umfeld eine Rolle, so sagt er, dass eine gute dörfliche Gemeinschaft, die Annahme von externen Hilfsangeboten unnötig macht, da sie Unterstützung und Halt geben kann.

\section{Kategorie Hilfsangebote}

Alle 5 informell Pflegenden treffen Aussagen zu einem psychoonkologischen Hilfsangebot. Vier stehen einem solchem Angebot eher ablehnend gegenüber, weil sie negative Vorerfahrungen im Familienkreis mit dieser Form von Angebot gemacht haben. Weiter können diese sich nicht vorstellen, mit welchen Themen und Inhalten ein Angebot für sie passend sein könnte, sodass es als hilfreich empfunden wird. Ein Befragter gibt auch an, dass der Zeitpunkt, als ihm psychologische Hilfe angeboten wurde, sehr ungünstig war, da es seiner Frau gesundheitlich sehr schlecht ging 
und er sich in dieser Phase nicht darauf einlassen konnte. Ein Interviewpartner nimmt bereits regelmäßig Gesprächstermine bei einer Psychologin wahr und empfindet diese als hilfreich. Positive Vorerfahrungen haben es ihm leichter gemacht ein solches Angebot zu nutzen.

Eine systematische Beratung haben 2 der 5 Befragten bekommen. Beraten wurden hier die Betroffenen und der pflegende Angehörige. Thematisch befassten sich die Beratungen mit pflegerischen Hilfsmitteln oder Ernährung beim Erhalt einer Chemotherapie. Mehr Informationen haben sich die pflegenden Angehörigen zu Leistungen des Gesundheitssystems gewünscht, da diese von den Angehörigen nur unter Mühen recherchiert werden konnten.

Selbsthilfegruppen werden von den Befragten kritisch gesehen. Drei der Befragten sehen keine Notwendigkeit und Mehrwert im Besuch einer Selbsthilfegruppe. Bereits Mitglied in einer Selbsthilfegruppe war ein Gesprächsteilnehmer. Er besuchte als Betroffener eine onkologische Gruppe. Weitere konnte er dann, bedingt durch die Krebserkrankung seiner Frau, aus Zeitgründen nicht mehr besuchen. Die Teilnahme ist besonders erschwert, wenn diese in ländlichen Gebieten wohnen, oder aufgrund mangelnder Mobilität.

Alle Befragten nutzen Entlastungsdienste wie Pflegedienste oder SAPV-Teams. Die Dienste übernehmen Tätigkeiten im Haushalt oder sind dafür zuständig, Zusatznahrung zu verabreichen. Selten übernehmen sie pflegerische Aufgaben, z. B. Körperpflege. Diese Versorgung übernehmen die informell Pflegenden größtenteils selbst. Das SAPV-Team ist auch als Notfallkontakt hilfreich. Krankenhausaufenthalte des Betroffenen konnten durch gezielte Unterstützung des SAPV-Teams reduziert werden. Hilfe erfuhren die informell Pflegenden ebenso durch Einrichtung des Gesundheitssystems, wie Krankenkassen, die bei der Beantragung von Hilfsmitteln laut den informell Pflegenden unterstützend waren. Eine weitere Entlastung, besonders für die pflegenden Angehörige im ländlichen Raum, war es, dass ihre Hausärztin eine Pflegefachkraft Hausbesuche machen ließ. Sie verteilte vor Ort Rezepte und führte Routinetests durch, sodass die Betroffenen dafür nicht immer die Praxis aufsuchen mussten. Ergebnisse und Befunde wurden anschließend mit den Ärzten/Ärztinnen telefonisch oder persönlich besprochen.

\section{Zusammenfassung der Ergebnisse}

Der zweischritte Forschungsprozess hilft, die Ergebnisse entsprechend den Forschungsfragen $\mathrm{zu}$ fokussieren. Die Untersuchung zeigt, dass ein unterstützendes soziales Umfeld für die pflegenden Angehörigen wichtig ist. Sowohl informelle Netzwerke in der Nachbarschaft als auch formelle Versorgungsstrukturen, wie z.B. SAPV-Teams. Sie unterstützen nicht nur bei der Begleitung und Betreu- ung, sondern auch bei der Beschaffung von Informationen zu Hilfs- oder Unterstützungsangeboten. Die Befragten können Unterstützungs- und Hilfebedarfe nicht präzise benennen. Weiter ist ihnen der Mehrwert bestimmter Angebote, z. B. Selbsthilfegruppen oder psychoonkologischer Beratung, unklar. Es zeigte sich weiter, dass die Inanspruchnahme von Hilfeleistungen von den Befragten selbst als Schwäche ausgelegt wird.

\section{Diskussion}

Die vorliegende Untersuchung hatte das Ziel, subjektive Hilfebedarfe speziell von informell Pflegenden, die onkologische Patienten betreuen, zu elaborieren. Die Herangehensweise in zwei Forschungsschritten erwies sich hier als zielführend. Der erste Forschungsschritt, also die Befragung von Experten, diente einer Annäherung des Forschungsfeldes und einem Überblick der regionalen Hilfe- und Unterstützungsleistungen. Der zweite Forschungsschritt konnte die forschungsleitende Frage beantworten.

Die Hilfe aus dem sozialen Netzwerk der informell Pflegenden stellte sich in den Expertengesprächen und auch in den Interviews als wichtig heraus. Vor allem die pflegenden Angehörigen, die im ländlichen Raum wohnen, beschreiben Nachbarschaftshilfen oder Unterstützung durch z. B. zeitweise Übernahme der Betreuung des zu Pflegenden von Personen aus ihrem Umfeld als hilfreich. Dies bestätigt Ergebnisse einer Studie von Drossel (2018) zur Situation informell Pflegender im Raum Oberfranken. Deshalb fordert Drossel (2018) die Stärkung dieser Strukturen durch den Aufbau einer Engagement-Gesellschaft, die Ehrenamt und Eigeninitiative fördert. Aufgrund der demografischen Entwicklung kann das Gesundheitssystem den geforderten Unterstützungsbedarf nicht durch professionelle Hilfeleistungen abdecken. Es werden ehrenamtliche Helfer benötigt, Leute, die sich engagieren und füreinander da sind. Bezug nimmt er hierbei auf ein Modellprojekt im Emsland. Dort hat der Aufbau einer solchen Gesellschaft bereits begonnen, denn es werden hier beispielsweise kleinere handwerkliche Dienstleistungen oder Hilfen zur Mobilitätserhaltung im Alltag untereinander angeboten (Drossel 2018).

Die Informationen zu Unterstützungsleistungen erreichen informell Pflegende über informelle Netzwerke im sozialen Umfeld. Das Angebotsspektrum in Erfurt ist nach Aussage der Experten im Forschungsschritt 1 noch unübersichtlich. Hier können zentrale Pflegestützpunkt eine gute Ergänzung sein. In Thüringen existieren 3 Pflegestützpunkte in den Städten Jena, Nordhausen und Sondershausen. Sie stellen zentrale Informationsstellen für pflegende Angehörige dar und beraten $\mathrm{zu}$ allen Themen der pflegerischen Versorgung. Des Weiteren wird es den informell Pflegenden dort ermöglicht, Kontakt zu Unterstützungsdiensten 
aufzubauen, und Leitungen können vernetzt werden (Thüringer Ministerium für Arbeit, Soziales, Gesundheit, Frauen und Familie, 2019). Eine flächendeckende und gut erreichbare Verortung von Informationsstellen im Raum Erfurt kann die Informationsbeschaffung für die Angehörigen erleichtern. In den Befragungen wird dies auch von den informell Pflegenden thematisiert. Sie wünschen sich eine Bündelung der für sie relevanten Informationen und eine zeitnahe Bereitstellung dieser. Die Bereitschaft, dies in einem Stützpunkt zu tun, zeigt sich durch die Tendenz der Informationsbeschaffung aus den Einrichtungen des Gesundheitssystems. Aus Expertensicht würde die Schaffung flächendeckender Pflegestützpunkte v.a. der älteren Bevölkerung zugutekommen, da sie mit der Informationsbeschaffung über das Internet zumeist nicht vertraut und überfordert sind. Das zeigt auch die Nutzung der 2016 online geschalteten Krebs-App. Sie enthält Informationen zu Selbsthilfegruppen, niedergelassenen Onkologen und psychoonkologischen Angeboten im Freistaat (Pätzold 2017). In den Interviews erwähnt keiner der informell Pflegenden diese App als Informationsquelle. Auch die befragten Experten beschreiben die App nicht als vorrangigen $\mathrm{Zu}-$ gangsweg zu Unterstützungsleistungen. Sie enthält nur Angebote für die an Krebs Erkrankten selbst; Hilfen, die speziell an Angehörige gerichtet sind, findet man nicht. Könnten aber in der App ergänzt werden. Diese Zugangswege könnten über Inhalte von Hilfsangeboten informieren, denn oft fällt es den Angehörigen schwer, sich vorzustellen, welche Angebote ihnen helfen könnten, und wo diese ansetzen. Der Grund liegt auch darin, dass der Fokus der informell Pflegenden in der Pflegesituation vorrangig auf dem Erkrankten liegt. Eigene Bedürfnisse werden zurückgestellt. Die Experten in der Befragung bestätigen dies und beschreiben, dass pflegende Angehörige ihre eigene Hilfebedürftigkeit häufig zu spät erkennen und sie evtl. schon selbst erkrankt sind. Eine frühzeitige Einbindung der informell Pflegenden in psychoonkologische Angebote oder die Vernetzung mit einer Krebsberatungsstelle würde diesem Prozess vorbeugen. Götze et al. (2015) beschreiben dies auch in der Untersuchung zum Belastungserleben von pflegenden Angehörigen. Sie beschreiben, dass, je länger die Pflegezeit andauert, desto größer das Belastungserleben ist, das infolgedessen die psychische Gesundheit der informell Pflegenden beeinträchtigen kann (Götze et al. 2015). Das „Leitlinienprogramm Onkologie“ empfiehlt in diesem Zusammenhang, dass es Angehörigen möglich gemacht werden muss, ein wohnortnahes Unterstützungsangebot aufzusuchen (Deutsche Krebsgesellschaft 2014). Informell Pflegende werden als Mitbetroffene wahrgenommen und sollen zur Annahme von Hilfsangeboten ermutigt werden (Deutsche Krebsgesellschaft.; Deutsche Krebshilfe; AMWF, 2015). Die Diagnosestellung an sich ist für die nächsten Angehörigen eine Ausnahmesituation, die es zu bewältigen gilt (Huse-Kleinstoll 2013). Dies beschreiben auch die pflegenden Angehörigen in den Interviews. Es wird von einer wachsenden Unsicherheit, auftretenden Angstgefühlen und Hilflosigkeit gesprochen. Dennoch möchte man diese Gefühle dem Betroffenen nicht zeigen, und somit werden sie zurückgestellt, um für den Anderen stark sein zu können. Hier den informell Pflegenden Informationen zur Verfügung zu stellen, die sich mit ihrer eigenen Hilfsbedürftigkeit befassen, erfordert Fingerspitzengefühl. Häufig stoßen diese Angebote dann auf Ablehnung.

Die Initiative, nach Hilfsangeboten zu suchen, kommt weniger von den informell Pflegenden, sondern zumeist durch ihr soziales Umfeld, die eine Veränderung bei den Angehörigen feststellen. Ebenso kommen Impulse, sich Hilfe zu suchen, von den behandelnden Ärzten der informell Pflegenden. Rosenberger et al. (2012) stellten außerdem fest, dass die Bereitschaft der informell Pflegenden, ein solches Angebot anzunehmen, höher ist, wenn dies von ärztlicher Seite vorgeschlagen wird. Dies wird auch in dieser Untersuchung deutlich. Ein sinnvoller Ansatz könnte hier die Etablierung eines Informationskonzeptes sein, um informell Pflegenden schon frühzeitig unterstützende Möglichkeiten anzubieten.

\section{Limitation}

Die gewählten Methoden und deren Ergebnisse bieten eine gute Grundlage für weitere Forschungen. Das Ziel der Untersuchung, den subjektiven Hilfebedarf speziell von informell Pflegenden, die onkologische Patienten betreuen, darzustellen, konnte durch zwei Forschungsschritte erreicht werden. Die gewählte Methode im ersten Forschungsschritt war im Austausch mit den Experten eine gute Herangehensweise, um Informationen über die Arbeitsweise der von ihnen angebotenen Unterstützungsleistungen, zu erhalten. Im zweiten Forschungsschritt gelang keine Heterogenität des Sample. Die Vielfalt der zu erforschenden Personengruppe, z.B. hinsichtlich der Erstversorgung der Angehörigen im stationären oder im ambulanten Setting, wird nicht abgebildet. Fast alle Angehörigen wurden stationär onkologisch versorgt. Nur ein Tumorpatient wurde ambulant therapiert. Da der Ambulantisierungstrend immer weiter fortschreitet, wäre es wichtig, die Forschung in diesem Bereich auszuweiten, da Unterschiede in den Hilfebedarfen, z. B. in der Information, Beratung und Anleitung, vermutet werden. Ein weiterer Schritt, der zu einer Ideenaggregation und Handlungsempfehlungen führen kann, wurde aus forschungspragmatischen Gründen nicht durchgeführt, wäre aber wichtig und sinnvoll. Zur Absicherung der theoretischen Datensättigung wären im zweiten Forschungsschritt weitere Interviews sinnvoll gewesen. Eine Generalisierung 
der Aussagen ist nicht sinnvoll und diese müssen durch weitere Forschungen Untersuchungen abgesichert werden.

\section{Implikationen für die Praxis}

Vor dem Hintergrund eines stetig fortschreitenden Ambulantisierungsprozesses, der die weitere Verlagerung von stationären Therapieleistungen in den ambulanten Sektor verlagert, muss Homecare in den Fokus gestellt werden. Der Wunsch des Patienten nach möglichst langer Versorgung im eigenen häuslichen Umfeld ist zu berücksichtigen. Daher muss das Umfeld des Pflegeempfängers gestärkt werden, um bei den vielfältigen Anforderungen in der Phase einer onkologischen Erkrankung die Versorgung unterstützen zu können. Dies kann nur gelingen, indem man ein frühzeitiges auf die informell Pflegenden abgestimmtes Informationskonzept über Hilfsmöglichkeiten mit in die Angehörigenarbeit integriert. Idealtypisch konzentriert sich dieses auf wenige Personen oder Institutionen, z. B. aufsuchende Beratung oder Pflegestützpunkte, die dann umfassend informieren, beraten und anleiten können. Eine Erweiterung des Angebotsspektrums im Raum Erfurt muss erfolgen. Es bleibt zu hoffen, dass mit gesteigerter Information auch die Bereitschaft der informell Pflegenden an solchen Unterstützungsmöglichkeiten geweckt wird, bestehende Vorurteile abgebaut werden können und die Hilfs- und Unterstützungsangebote als nutzbringend bewertet werden. Das systematische Aufbauen einer Engagement-Gesellschaft, also eine Gesellschaft, die sich im Alltag unterstützt, z. B. durch Nachbarschaftshilfen, kann einen wichtigen weiteren Beitrag leisten. Diese müssen, besonders auch politisch, gefördert und weiter forciert werden. Denn nur so gelingt es pflegenden Angehörigen, die Belastungen, die während der Pflegezeit auftreten, zu reduzieren, selbst gesund zu bleiben, um so lange wie möglich für den Betroffenen da sein zu können. Ihnen dabei zu helfen, ist von großer Relevanz, denn sie stellen eine der wichtigsten Stützen unseres Gesundheitssystems dar. Die professionelle Pflegeausbildung, z.B. durch ,,advanced practice nursing“, kann einen weiteren wichtigen Beitrag zu Gemeinde- und Familienpflege leisten.

Funding Open Access funding enabled and organized by Projekt DEAL.

Interessenkonflikt M. Drossel und D. Sperling geben an, dass kein Interessenkonflikt besteht.

Open Access Dieser Artikel wird unter der Creative Commons Namensnennung 4.0 International Lizenz veröffentlicht, welche die Nutzung, Vervielfältigung, Bearbeitung, Verbreitung und Wiedergabe in jeglichem Medium und Format erlaubt, sofern Sie den/die ursprünglichen Autor(en) und die Quelle ordnungsgemäß nennen, einen Link zur Creative Commons Lizenz beifügen und angeben, ob Änderungen vorgenommen wurden.
Die in diesem Artikel enthaltenen Bilder und sonstiges Drittmaterial unterliegen ebenfalls der genannten Creative Commons Lizenz, sofern sich aus der Abbildungslegende nichts anderes ergibt. Sofern das betreffende Material nicht unter der genannten Creative Commons Lizenz steht und die betreffende Handlung nicht nach gesetzlichen Vorschriften erlaubt ist, ist für die oben aufgeführten Weiterverwendungen des Materials die Einwilligung des jeweiligen Rechteinhabers einzuholen.

Weitere Details zur Lizenz entnehmen Sie bitte der Lizenzinformation auf http://creativecommons.org/licenses/by/4.0/deed.de.

\section{Literatur}

Beckmann M, Adler G, Albers P, Bruns J, Ehninger G, Hauschild A et al (2008) 3-Stufen Modell optimiert Behandlung unter Kostendeckung - Wie die künftigen Strukturen der onkologischen Versorgung in Deutschland aussehen sollten. Geburtshilfe Frauenheilkd 68:658-661

Corbin J, Hildebrand B (2003) Qualitative Forschung. In: Rennen-Allhof B, Schaeffer D (Hrsg) Handbuch Pflegewissenschaft. Juventa, Weinheim, München

Deutsche Krebsgesellschaft, Deutsche Krebshilfe, AWMF (2014) Leitlinienprogramm Onkologie Psychoonkologische Diagnostik, Beratung und Behandlung von erwachsenen Krebspatienten. http:// leitlinienprogramm-onkologie.de/Leitlinien.7.0.html (Erstellt: 1. Jan. 2014). Zugegriffen: 28. Okt. 2018 (AWMF-Registernummer: 032/051OL)

Deutsche Krebsgesellschaft, Deutsche Krebshilfe, AWMF (2015) Leitlinienprogamm Onkologie Palliativmedizin für Patienten mit einer nicht heilbaren Krebserkrankung. http://leitlinienprogrammonkologie.de/Palliativmedizin.80.0.html (Erstellt: 1. Jan. 2015). Zugegriffen: 28. Okt. 2018 (AWMF-Registernummer: 128/001OL)

Deutsches Krebsforschungszentrum (2017) Fragen zu Krebs? https:// www.krebsinformationsdienst.de/grundlagen/krebsstatistiken. php\#inhalt2. Zugegriffen: 28. Okt. 2018

Dresing T, Pehl T (2018) Praxisbuch Interview, Transkription \& Analyse. Anleitungen und Regelsysteme für qualtitativ Forschende. Eigenverlag, Marburg

Drossel M (2018) Versorgung älterer Menschen durch die Stärkung informeller Pflege Am Beispiel der Region Oberfranken. Jacobs, Lage

Göbel H (2017) Bericht von der Mitgliederversammlung des Tumorzentrums Erfurt e.V. am 06.09.2017. J Tumorzent Erfurt (01):28-31

Götze H, Brähler E, Gansera L, Schnabel A, Köhler N (2015) Erschöpfung und Überlastung pflegender Angehöriger von krebspatienten in der palliativen Situation. Psychother Psychosom Med Psychol 65:66-72

Helfferich C (2009) Die Qualität qualitativer Daten Manual für die Durchführung qualitativer Interviews. VS, Wiesbaden

Huse-Kleinstoll G (2013) Psychoonkologische Arbeit mit Familien. In: Schulz-Kindermann F (Hrsg) Psychoonkologie Grundlagen und psychotherapeutische Praxis. Beltz, Weinheim, Basel, S 188-199

Kuckartz U (2012) Qualitative Inhaltsanalyse. Methoden, Praxis, Computerunterstützung. Beltz Juventa, Weinheim, Basel

Mayring P (2015) Qualitative Inhaltsanalyse. Beltz, Weinheim, Basel

Meuser M, Nagel U (1991) ExpertInneninterviews - vielfach erprobt, wenig bedacht: ein Beitrag zur qualitativen Methodendiskussion. Westdeutscher Verlag, Opladen. https://www.ssoar.info/ssoar/ handle/document/2402

Pätzold E (2017) Entwicklung Krebs-APP in Thüringen für Betroffene und deren Angehörige. Brückenschlag. https://de.readkong.com/ page/bruckenschlag-das-erfurter-selbsthilfemagazin-ausgabe2017-9271172

von Reibnitz C (2008) Homecareversorgung. In: Bäumer R, Maiwald A (Hrsg) Onkologische Pflege. Thieme, Stuttgart, S 12-17 
Robert Koch Institut (2016) Bericht zum Krebsgeschehen in Deutschland 2016. Robert Koch Institut, Berlin

Rosenberger C (2017) Trends in der ambulanten Versorgung. Nervenheilkunde 36:963-968

Rosenberger C, Höcker A, Cartus M, Schulz-Kindermann F, Härter M, Mehnert A (2012) Angehörige und Patienten in der ambulanten psychoonkologischen Versorgung: Zugangswege, psychische Belastungen und Unterstützungsbedürfnisse. Psychol Psychosom Medzinische Psychol. https://doi.org/10.1055/s-0032-1304994

Stoll H, Schmidt S (2011) Häusliche Betreuung und Pflege von Tumorpatienten. In: Margulies A, Kroner T, Gaisser A, Bachmann-Mettler I (Hrsg) Onkolgische Krankenpflege. Springer, Berlin, Heildberg, S 701-710
Thüringer Ministerium für Arbeit, Soziales, Gesundheit, Frauen und Familie (2019) Soziales. https://www.thueringen.de/th7/tmasgff/ soziales/pflegestuetzpunkte/. Zugegriffen: 13. Febr. 2019

Weihs K, Reiss D (2000) Family reorganization in response to cancer: a developmental perspective. In: Baider L, Cooper CL, Kaplan De-Nour A (Hrsg) Cancer and the family, 2. Aufl. John Wiley \& Sons Ltd, Chichester, S 17-39

Hinweis des Verlags Der Verlag bleibt in Hinblick auf geografische Zuordnungen und Gebietsbezeichnungen in veröffentlichten Karten und Institutsadressen neutral. 